\title{
STELLAR CONVECTION THEORY
}

Jean-Paul Zahn

Observatoire de Nice

06300 Nice, France

\section{INTRODUCTION}

This meeting, which dea1s with turbulence in stars, opens with a review on thermal convection. There is no better way to state from the start that among all instabilities that are likely to arise in stars, it is thermal convection which is the most firmly established as a cause for the turbulence that we observe on their surface. Our confidence in this comes mainly from the theoretical prediction that convective instability sets in whenever the density stratification becomes superadiabatic, as is expected in late type stars whose outer layers are very opaque, due to the ionization of the two most abundant elements, hydrogen and helium. And, in these stars at least, thermal convection occurs close enough to the photosphere to influence, be it indirectly, the profile of spectral lines.

A whole IAU colloquium has been devoted three years ago in Nice to the topic of stellar convection, and one finds in its proceedings an extensive account of what was the state of the problem. Some progress has been accomplished since then, and natura1ly I will spend most of my time describing recent work, and even work in progess that $I$ an aware of. But on the assumption that some of you are not too familiar with the subject, let me first recall some generalities.

\section{A HIGHLY NONLINEAR PROBLEM}

Thermal convection is described by a set of we11-known equations, which state the conservation of mass

$$
\frac{\partial \rho}{\partial t}+\nabla \cdot \rho \underline{V}=0,
$$

that of momentum

$$
\rho\left(\frac{\partial \underline{V}}{\partial t}+(\underline{V} \nabla) \underline{V}\right)=-\nabla P+\underline{g} \rho+\underline{f}-V
$$


and that of heat (or entropy)

$$
\rho T\left(\frac{\partial S}{\partial t}+\underline{v} \cdot \nabla S\right)=\nabla \cdot(K \nabla T)+d_{V}
$$

One can combine these equations to establish the conservation of total energy

$$
\begin{gathered}
\frac{\partial}{\partial t}\left(\frac{1}{2} \rho \mathrm{V}^{2}+\rho \mathrm{H}-\mathrm{P}\right)+\nabla \cdot\left(-\mathrm{K} \nabla \mathrm{T}+\mathrm{H} \rho \underline{\mathrm{V}}+\frac{1}{2} \rho \mathrm{V}^{2} \underline{\mathrm{V}}+\underline{\mathrm{F}} \mathrm{V}\right) \\
=\underline{g} \cdot \rho \underline{\mathrm{V}}
\end{gathered}
$$

I use here the classical notations for the gravity vector ( $g$ ) and the velocity field $(\underline{V})$; the pressure ( P ), the entropy ( S ), the enthalpy $(\mathrm{H})$ and the thermal conductivity $(K)$ are all known functions of the density $(p)$ and the temperature $(T)$. The viscous force $\left(\underline{f}_{v}\right)$, the viscous dissipation $\left(d_{v}\right)$ and the so-called viscous flux $\left(\underline{F}_{v}\right)$ need not to be explicited for this purpose. The assumption has been made that the medium is optically thick, so that the radiative flux is proportional to the temperature gradient; $\mathrm{H} \rho \mathrm{V}$ is the convective flux, and $1 / 2 \rho \mathrm{V}^{2} \underline{\mathrm{V}}$ the mechanica1 energy flux.

Even a layman realizes that the differential system formed by the first three equations is highly nonlinear, and he may guess that most difficulties in treating the problem are due to this nonlinearity. To stress that point, it suffices perhaps to recall that the most simple version of this system obtained by neglecting the buoyancy and assuming that the density is constant, and thus retaining only the two first equations (which the fluid dynamicists refer to as the Navier-Stokes equations), has no general solution that would be valid over a large range of parameters.

The nonlinearities of the problem have a double role. First they prevent the motions, once the instability has set in, from growing for ever exponentially. Second, they generate a whole set of scales in the velocity field, and they interconnect them. The term responsible for this is the advection term $(\underline{V} \nabla) \underline{v}$, and no wonder that this term, plus a similar one in the heat equation, namely $\underline{V} \nabla S$, are the most difficult to deal with when attempting to solve the problem numerically.

In fact, the various approaches to the problem that have been deviad so far differ mainly in how they treat these advection terms. The most ambitious approach would be of course to consider al1 scales, and to calculate explicitly those nonlinear terms. However in all cases of astrophysical interest, this is out of question since the scales involved span several orders of magnitude (typically eight or more), and even if the velocity field were homogeneous in space, which it is not, describing all these scales would be well beyond the capabilities of the present computers. Therefore one has always, at one stade or another, to make some simplifying assumption. An impressive portion of the fluid dynamical literature has been devoted to the subject of how to best model convective transport, that is the advection, by prescribed motions, of a scalar or of a vector field. Let us briefly recal1 how this is done most commonly. 
3. APPROXIMATING THE CONVECTIVE TRANSPORT

The simplest situation one can imagine is that of the advection of a passive scalar, ignoring molecular diffusion. The conservation equation for this scalar (which may be a dye in water, a pollutant in the atmosphere or entropy in thermal convection) takes the simple form

$$
\frac{\partial s}{\partial t}+\underline{v} \cdot \nabla s \quad \Rightarrow \quad 0
$$

$s$ being the concentration.

Experiments and observations show that in many instances, in particular when the motions are turbulent, the scalar just diffuses away. Thus in that case some local average of $\mathrm{s}$, which we will designate by $\vec{s}$ without further defining it, obeys a diffusion law

$$
\frac{\partial \bar{s}}{\partial t}-\nabla \cdot\left(x_{\mathrm{T}} \nabla \bar{s}\right)=0
$$

The turbulent diffusivity $x_{T}$ is given by

$$
x_{\mathrm{T}}=z_{\mathrm{u}}
$$

$\mathrm{u}$ being the mean turbulent velocity and $z$ the mixing zength. The latter has been introduced by Prandtl (1925), and can be viewed as the distance a turbulent element travels before it dissolves in the medium and dumps there the scalar quantity that it has carried to that point.

For this diffusion approximation to be valid, a necessary condition is obviously that the mixing length be smaller than the scale characterizing the spatial variation of $\bar{s}$. Unfortunately, this is not a sufficient condition, as it has been recognized in many cases.

The most recent work on this subject is that by Knobloch (1978), who begins to establish a formal expression for the time derivative of $\bar{s}$

$$
\frac{\partial \bar{s}}{\partial t}=\left(\sum_{m=2}^{\infty} K_{m}\right) \bar{s}
$$

The $\mathcal{K}_{\mathrm{m}}$ are differential operators which can in principle be determined for a given velocity field. If this field is isotropic and varies slowly enough in space and time, the $m$ are given by

$$
K_{m}=n_{2}^{m} \nabla^{2}+n_{4}^{m} \nabla^{4}+\cdots+n_{m}^{m} \nabla^{m^{\prime}}
$$

(where $\mathrm{m}^{\prime}$, an even number, is either $\mathrm{m}$ or $\mathrm{m}-1$ ). The coefficients $\eta_{i}^{m}$ are integrals involving the velocity distribution and its successive moments.

For $\mathrm{m}=2$, one recovers the above mentioned diffusion approximation, with $\begin{array}{r}2 \\ \eta_{2}\end{array}$ being the turbulent diffusivity. For higher $m$, the coefficient in front of the Laplacian operator becomes

$$
n_{2}^{2}+n_{2}^{3}+n_{2}^{4}+\cdots
$$

and thus the diffusivity is modified, even when the higher order operators play a 
negligible role. The important fact to notice therefore is that at each step of approximation, as m increases, the coefficient multiplying each differential operator is renormalized.

Moreover, the series is known only to converge if the following dimensionless number is smaller than unity: $u_{\tau} / \lambda<1$, where $\tau$ and $\lambda$ are respectively the correlation time and the correlation length characterizing the velocity field (see Knobloch's paper for more detai1s). In most cases however that requirement is not met, and this restricts seriously the applicability of the diffusion approximation. Needless to say that the problem becomes even more intricate if it is a vector field that is being advected, if it feeds back on the velocity field, if the latter is no longer isotropic, etc.

At this point we realize that the only reason the diffusion approximation is so widely used is that one has not yet found by what to replace it. And we come to understand that the degree of refinement (I hesitate to say reliability) of a given treatment of thermal convection can be measured by the level at which the diffusion approximation is introduced, as we will see next.

\section{THE MIXING LENGTH APPROACH}

In the current mixing length approach, which has been applied to stellar convection (in the core of a star) by Biermann (1933), the diffusion approximation is used right from the beginning to model the heat transport. Thus the advection term in Eq. 3, $\rho \mathrm{T} \underline{\mathrm{V}} \cdot \nabla \mathrm{S}$, is replaced by

$$
-\nabla \cdot\left(x_{\mathrm{T}} \rho \mathrm{T} \nabla \mathrm{S}\right)=-\nabla \cdot\left(\underline{\mathrm{F}}_{\mathrm{C}}\right)
$$

the quantity in parenthesis being the convective flux, whose magnitude is more commonly stated as

$$
F_{C}=\rho C_{P} T(u \quad Z / H)\left(\nabla_{\text {ad }}-\nabla\right)
$$

Here $C_{P}$ is the specific heat at constant pressure and $H$ the pressure scale height, $\nabla_{\text {ad }}$ and $\nabla$ are respectively the adiabatic and the true logarithmic temperature gradient, with respect to the pressure. The turbulent diffusivity has been replaced by u $l$, where usually the mixing length $Z$ is taken proportionaly to the pressure scale height and the velocity $u$ estimated from the conservation of mechanical energy:

$$
\frac{1}{2} \rho \mathrm{v}^{2} \sim \mathrm{g} \rho^{\prime} z
$$

The density difference $\rho$ ' between a convective element and its surroundings is evaluated in turn through

$$
\rho^{\prime} / \rho \sim\left(\nabla_{\text {ad }}-\nabla\right) \quad \tau / \mathrm{H}
$$

All quantities are then defined at any given depth, and the temperature gradient, for instance, depends only on those local values. 
Many improvements have been brought to this original form of the mixing length treatment, for instance by öpik (1950). Vitense (1953) took into account the heat exchanges between the convective elements and the surrounding medium, which enabled her to model a convection zone up to the surface of a star, thus linking its atmosphere with the interior. More recently Shaviv and Salpeter (1973), followed by Maeder (1975), endeavoured to transform the local mixing length treatment into a non-local one, which would be capable of also describing penetrative convection. Another line of action has been to refine the evaluation of the diffusion coefficient $\chi_{T}$, by introducing the effect of rotation and magnetic field; I will mention here only the most recent contributions from Gough (1978) and from Durney and Spruit (1979), since P. Gilman will cover this subject in more detail in his review.

Al1 these improvements however cannot hide the main weakness of the mixing length approach, namely that it evluates the heat transport through the diffusion approximation. Some progress should therefore be expected when abandoning this approximation, as it is done in the so-called hydrodynamical treatments.

\section{HYDRODYNAMICAL APPROACHES}

In such approaches, a great effort is made to solve the original fluid dynamical equations, at least for those scales of the velocity field that transport most of the convective flux. The diffusion approximation cannot be avoided entirely, but it will be used only to evaluate the momentum transport by the smaller scales. This is possible in principle if only the larger scales are driven by buoyancy, the smaller scales being generated through the nonlinear interactions that have been mentioned earlier.

By large, we mean here the scales that in the laboratory are of the order of the thickness of the unstable layer. In a star, several of such scales seem to be present, as indicated by the solar observations: those of granulation and supergranulation are well established, but others may also exist, as we will learn from J. Beckers later in this meeting. These scales are certainly related to the vertical structure of the convection zone, although we do not know yet precisely how, especially when the hydrogen and helium ionization regions are merged in the same unstable zone.

The numerical techniques available to solve the nonlinear system (Eq. 1-3) fall into three categories. First, you may choose to stay in the physical space; you divide it in a mesh as fine as your computer allows it, you transform the differential equations in finite differences equations, and you solve those by an appropriate scheme. Its transparency makes this method very appealing, but it remains restricted to rather mild convection, which does not require too high spatial resolution. Alternatively, since you are not interested in each detail of the various fields, but merely in their statistical properties, you might prefer to work in the Fourier space. This is done indeed by most people who study developed turbulence, but the method is only well suited 
for homogeneous fields.

Most calculations of stellar convection are based on a third method, which combines the main advantages of the other two. Postulating, as it seems very natural, that the various fields are homogeneous on horizontal surfaces in some statistical sense, you take a Fourier expansion (or something similar) in the horizontal directions, but you stay in the physical space for the vertical dimension, in which you are certain to encounter much more structure. This procedure is known as that of modal expansion; let us sketch it below when applied to a scalar field, say the temperature:

$$
\mathrm{T}(\mathrm{x}, \mathrm{y}, \mathrm{z}, \mathrm{t})=\overline{\mathrm{T}}(\mathrm{z}, \mathrm{t})+\sum_{i} \mathrm{f}_{i}(\mathrm{x}, \mathrm{y}) \theta_{i}(\mathrm{z}, \mathrm{t})
$$

The temperature is split into its horizontal average $\bar{T}$, which depends only on the vertical coordinate $z$ and time $t$, and the fluctuation around this mean, which is expanded into a series of horizontal planforms $f_{i}(x, y)$, the modal amplitudes $\theta_{i}$ being again functions of only $z$ and $t$.

The horizontal planforms used most commonly, mainly for simplicity, are those which separate the variables in the linear limit of the problem; they obey the harmonic equation

$$
\left(\frac{\partial^{2}}{\partial x} 2+\frac{\partial^{2}}{\partial y} 2\right) f_{i}+a_{i}^{2} f_{i}=0
$$

where $a_{i}$ is the wavenumber that characterizes the horizontal structure of this mode. Fourier modes of the same wavenumber can be assembled to describe cells of various shapes: horizontal rolls, prisms of rectangular base, of hexagonal base, etc., which may differ stronlgy in their nonlinear properties.

Many other possible choices exist for these planform functions; one has recently been explored by Depassier and Spiegel (1979), which needs only one single mode to render the high contrast that is often observed between rising and falling motions.

\section{RECENT DEVELOPMENTS}

The modal expansion procedure has first been applied to the Boussinesq approximation, which is the form the fluid dynamics equations take when the thickness of the considered layer is very small compared to the density scale height, as it is the case in laboratory convection. In this approximation, the only nonlinear terms are - not surprisingly - the advection terms $(\underline{V} \nabla) \underline{V}$ and $\underline{V} \cdot \nabla S$, and much can be learned by studying this simplest example of thermal convection. The parameters defining a given experiment reduce then to only two: the Rayleigh number, which measures the strength of the instability, and the Prandtl number, which is the ratio of the viscosity to the thermal diffusivity. It is therefore relatively simple, in principle, to explore the behavior of convection, both experimentally and theoretically, in the two-dimensional space of these parameters.

For a thorough discussion of Boussinesq convection we refer to the review paper 
by Spiegel (1971). The most extensive calculations, with the modal expansion procedure, have been performed by Toomre et al. (1977); their results are in satisfactory agreement with the laboratory experiments. One difficulty with the modal method is the selection of the planforms that are used in the expansion, especially when the available facilities limit the number of modes to one or two only. Numerical experimentation of the kind just quoted then offers an indispensable guidance on how to make such choices.

\section{a. Strong density stratification}

The Boussinesq approximation is of course not well adapted to describe stellar convection, which takes place in general over several density scale heights. Therefore some of the recent works have dealt with more realistic situations and have focussed on the effects due to a strong density stratification. Another approximation is then used very often, namely the anelastic approximation. It consist mainly in filtering out the acoustic waves, which probably contribute very little to the energy transport. To be applied, it requires that the convective velocities be small compared to the speed of sound (see Gough 1969), which is generally the case.

The simplest example to consider is that of a thick layer of perfect gas, in which the dynamical viscosity and the thermal conductivity are both constant. In the absence of motion, the stratification is that of a polytrope, with $\ln \rho=\mathfrak{n} \ln T+$ constant. As is well known, the stratification is convectively unstable when $n$, the polytropic index, is less then 1.5 (for either a monoatomic or a fully ionized gas).

The linear stability problem has been investigated by Spiegel (1965) and by Gough et al. (1976); steady nonlinear solutions have been constructed by Massaguer and Zahn (1979). The main result of those studies is that the pressure forces play a very important role, in contrast with Boussinesq convection, and the reasons for this can be easily understood.

In the anelastic approximation, the equation of momentum conservation can be written

$$
\bar{\rho}\left(\frac{\partial V}{\partial t}+(\underline{V} \nabla) \underline{V}\right)=-\nabla P^{\prime}+g \rho^{\prime}+\underline{f} V
$$

where $P^{\prime}$ and $\rho^{\prime}$ are the fluctuating values of the pressure and the density, with respect to their horizontal mean (of which only that of the density, $\bar{\rho}$, enters explicitly in this equation). As before in Eq. 1, $\underline{f}_{\mathrm{v}}$ is the viscous force. One sees that the pressure force, on the right hand side, has been split in the contribution of the fluctuating pressure, $-\nabla \mathrm{P}^{\prime}$, and that of the mean pressure, $\mathrm{g}^{\prime}$, which is usually referred to as the buoyancy force.

In Boussinesq convection, the buoyancy force is always acting upwards on a rising fluid element in the unstable region. A first consequence of the density stratification is that the buoyancy changes sign in the upper part of the unstable domain. To under- 
Eigure i

A cross-section through steady cellular convection

a. The pressure fluctuations are positive where the flow diverges, negative where it converges.

$b$ and c. Variation of the relative fluctuations of the pressure $\left(P^{\prime} / \bar{P}\right)$, the temperature $\left(\mathrm{T}^{\prime} / \overline{\mathrm{T}}\right)$ and the density $\left(\rho^{\prime} / \bar{p}\right)$, along the vertical ine A B, where the fluid is rising.

b. In the limit of vanishing density stratification (Boussinesq case), $P^{\prime} / \bar{P}$ is negligible and thus $\rho^{\prime} / \bar{\rho}$ is the mirror image of $T^{\prime} / \overline{\mathrm{T}}$.

c. With a strong stratification, $\mathrm{P}^{\prime} / \overline{\mathrm{P}}$ is of the same order as the other fluctuations, and therefore $\rho^{\prime} / \bar{\rho}$ (and thus the buoyancy force) changes sign in the upper part of the domain.

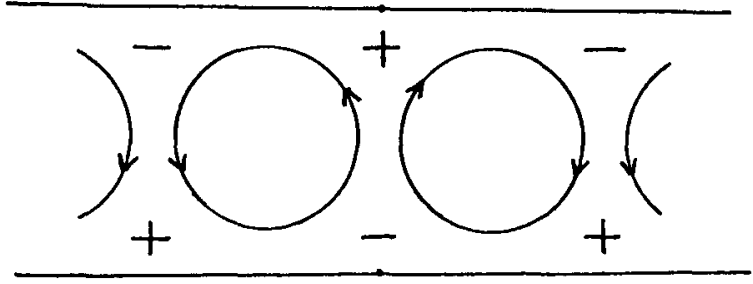

A

B

b. Boussinesq

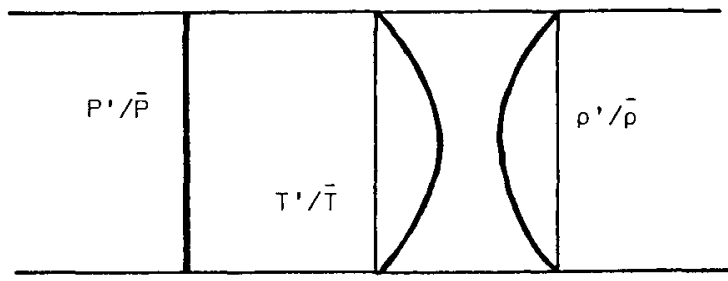

A

B

c. Stratified

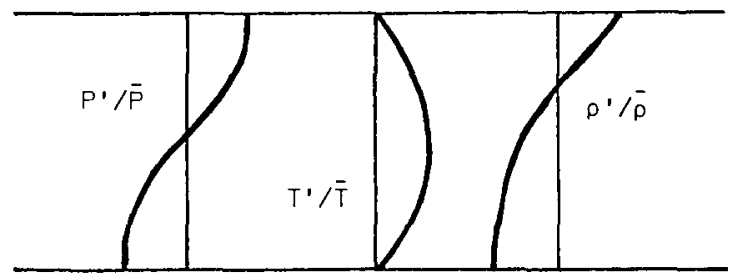

A

stand this, let us consider Figure la where we have sketched a cross-section through a convective layer. It makes no difference here whether the cells are rolls or take another shape: since it is the fluctuating pressure which is responsible for turning a vertical flow into a horizontal one, it must have a maximum in each horizontal plane where the flow diverges, and a minimum where it converges. This obliges the vertical gradient of the fluctuating pressure to be predominantly in the direction of the vertical flow, as depicted in Figure 1c. The temperature fluctuations, on the other hand, are positively correlated with the vertical velocities, since they originate from the advection of heat in a superadiabatic temperature gradient (in the language of the mixing length theory: a rising fluid element is hotter than its surroundings).

Now in Boussinesq convection the pressure fluctuations are vanishingly smal1 compared to the temperature fluctuations: the equation of state therefore imposes that the density fluctuations then just be the mirror images of these temperature fluctua- 
tions, as shown in Figure 1b. But in stratified convection, where the fluctuations of the pressure may be of the same order as those of the temperature (depending on the thickness of the convective layer as compared to the density. scale height), the buoyancy force reflects the horizontal variations of both the temperature and the pressure, as indicated in Figure $1 c$. This is again imposed by the equation of state, which in the anelastic approximation takes the simple linear form

$$
\mathrm{P}^{\prime} / \overline{\mathrm{P}}=\rho^{\prime} / \overline{\mathrm{\rho}}+\mathrm{T}^{\prime} / \overline{\mathrm{T}}
$$

with our assumption of a perfect gas. The profiles sketched in Figure 1 are valid for non penetrating convection, with the temperature fluctuations vanishing at top and bottom, but the results can easily be extended to other cases.

Another consequence of strong density stratification is the modification of the energetics of thermal convection. The work done by the fluctuating pressure force, per unit time and over the whole considered domain, is

$$
E_{P}=-\iiint \nabla P^{\prime} \cdot \underline{v} d v,
$$

which can be transformed into

$$
\mathrm{E}_{\mathrm{P}}=-\iint \nabla \cdot\left(\mathrm{P}^{\prime} \underline{\mathrm{v}}\right) \mathrm{ds}+\iiint \mathrm{P}^{\prime} \nabla \cdot \underline{\mathrm{V}} \mathrm{dv} .
$$

With the usual boundary conditions on $\underline{v}$, the surface integral is zero and only remains the volume integral, which also vanishes in the Boussinesq limit since there $\nabla \cdot \underline{V}=0$. But one sees that in a stratified medium, where the conservation of mass imposes that $\nabla \cdot \underline{v} \neq 0$, the fluctuating pressure too contributes to the work done on the fluid. There are many cases, as shown by Massaguer and Zahn (1979), where this pressure integral is larger than that representing the work done by the buoyancy force; there are instances where the latter work becomes even negative, due to the reversal of the buoyancy that has been discussed above.

We should perhaps remind ourselves at this point that in the mixing length approach the pressure fluctuations are generally ignored and that the convective velocities are estimated from the work done solely by the buoyancy. The application of this procedure should therefore be restricted to the Boussinesq limit, where the pressure fluctuations are indeed negligible and on1y the buoyancy force produces net work. But the mixing length approach is widely used to describe very thick convection zones, where these conditions are no longer realized, and this is another reason for not taking its predictions too seriously.

The properties of non Boussinesq convection that have been presented above in the case of a polytropic atmosphere are entirely confirmed by more realistic calculations, such as those performed by Latour, Toomre and Zahn with application to the envelope of an A type star; a first report on this has been given in Toomre et al. (1977). The medium is treated as a real gas, the variations of the thermal conductivity and of the turbulent viscosity are taken in account. The computational domain goes from above the photosphere to a depth of $28,000 \mathrm{~km}$, thus encompassing both convection zones (that 
due to the ionization of hydrogen and the first ionization of helium, and that due to the second ionization of helium); the density varies by a factor of 60 between top and bottom. The star has an effective temperature of $8000 \mathrm{~K}$ and a gravity of $1.1510^{4} \mathrm{~cm} \mathrm{~s}$.

A typical solution is displayed in Figure 2 for one choice of the horizonta1 planform of the single mode that has been retained in this calculation: it corresponds to cells of hexagonal base with the flow directed mainly upwards along their centerline. The horizontal size of the cell is comparable to the thickness of the lower convection zone. Notice that the pressure fluctuations are of the same order as those of the temperature; as in the simple polytrope, the buoyancy force is controlled by the pressure fluctuations in the upper part of the domain, whereas the temperature fluctuations dominate in the lower part. One verifies also that the fluctuations of these thermodynamical variables do not exceed $8 \%$, which justifies a posteriori the use of the anelastic approximation, in which only linear terms in these variables are retained.

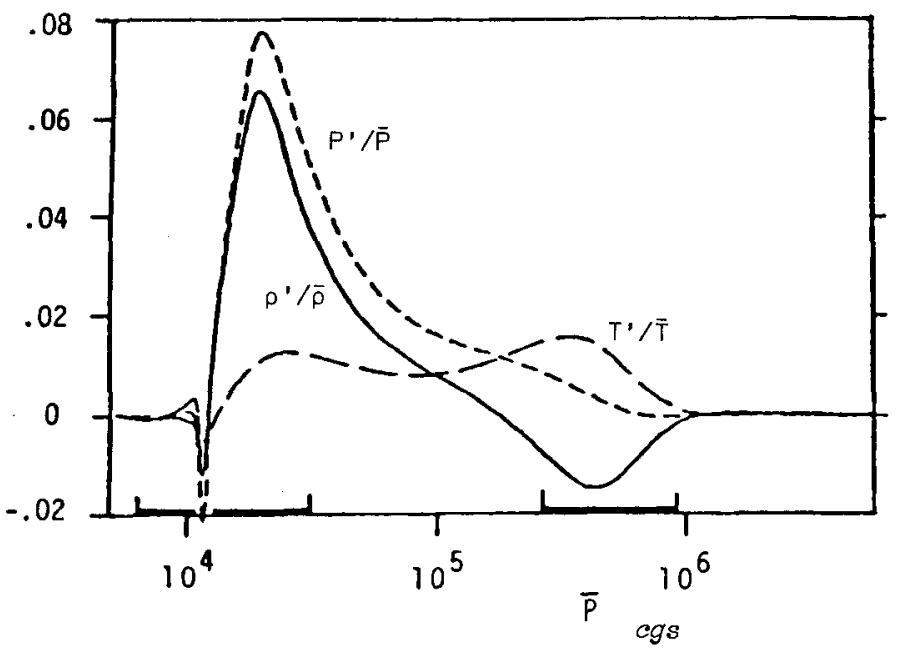

Figure 2

Fluctuations of the thermodynamical variables in the envelope of an A type star

The relative fluctuations of the pressure $\left(P^{\prime} / \bar{P}\right)$, the temperature $\left(\mathrm{T}^{\prime} / \overline{\mathrm{T}}\right)$ and the density $\left(\rho^{\prime} / \bar{\rho}\right)$ are shown versus the mean pressure, taken as measure of the depth. The location of the unstable zones is indicated in heavy lines on the pressure scale. The results refer to a single mode solution, and the fluctuations which are displayed are the modal amplitudes. The horizontal planform is hexagonal and the vertical velocity is predominantly upwards in the centerline of the convective cells, whose horizontal dimension is of the order of the thickness of the lower convection zone. 


\section{b. Penetration}

But the most striking result of this calculation is that the convective motions, which are driven in the lower convection zone, penetrate through the stable region above and the upper convection zone up to the surface of the star. This appears clear1y in Figure 3, where the two components of the velocity are shown for the same single mode solution as above. The strong horizontal flows which develop in the atmosphere are organized in cells whose dimensions are large compared to the local density or pressure scale heights; one is therefore tempted to identify this with some kind of supergranulation. In an earlier work by Toomre et al. (1976), there was already an indication for such an extended penetration, which has the consequence of connecting the two convection zones; according to the current mixing length theory, these zones should be well separated.

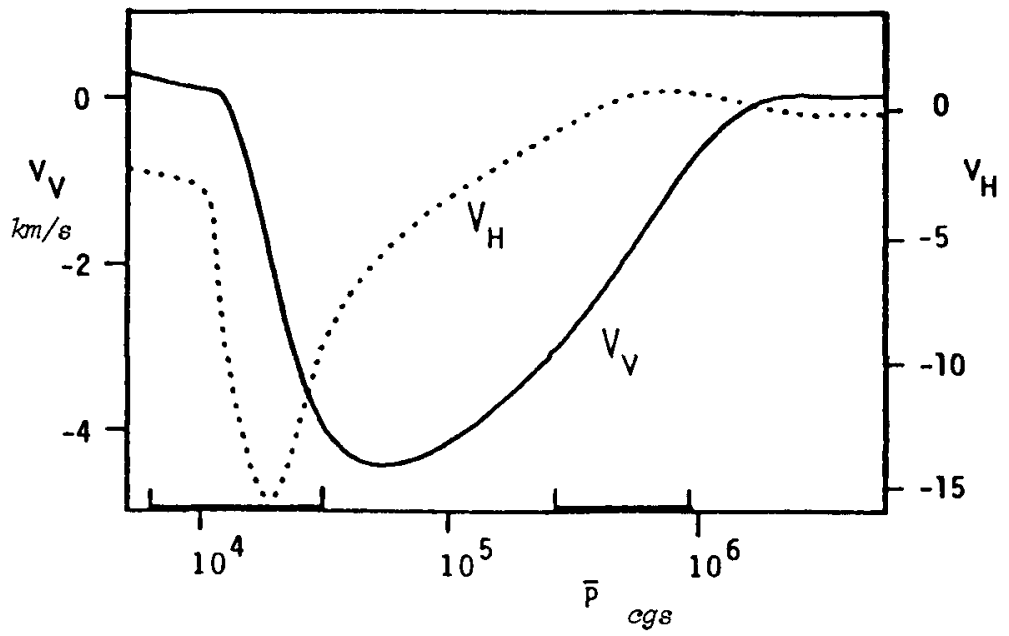

Figure 3

Convective velocities in the envelope of an A type star

The modal amplitudes of the vertical and horizontal velocities, designated respectively by $V_{V}$ and $V_{H}$, as functions of the mean pressure. This single mode solution is the same as that of Figure 2 . Over most of the domain, the vertical velocity has a negative sign, implying that it is directed upwards in the centerline of the convective cells. Notice the ample penetration from the lower convection zone all the way up to the surface of the star, and also the large horizontal velocities which occur there. 
Convection in smaller scales has not been investigated in this calculation, because the radiative transfer has been treated for simplicity in the diffusive approximation, which is not applicable in the optically thin 1imit. Nelson and Musman (1977) and Nelson (1978a) have tackled this problem, with application to the solar granulation. They treat the transfer in the Eddington approximation, whose validity has been established by Unno and Spiegel (1966) (at least when the mean radiation field is uniform). The mean stratification is imposed, and the amplitude of the convective motions is limited through a nonlinear process which is similar to a turbulent viscosity. Only the upper part of the solar convection zone is considered, above optical depth $\tau=25$; although the boundary conditions on the fluctuating variables appear somewhat arbitrary, their influence on the solutions seems moderate. The free parameters are chosen to match the observations.

The main results of these calculations concern the temperature fluctuations, which are shown to be controlled mainly by the transfer of radiative energy. They change sign around $110 \mathrm{~km}$ above optical depth $\tau=1$, as the result of penetrative convection, which has an e-folding distance of about $160 \mathrm{~km}$. The center to limb variation of the intensity fluctuations are in satisfactory agreement with the observations.

The same method has also been applied by Ne1son (1978b) to an F type star, which has two separate convection zones. His conclusions are similar to those of Latour, Toomre and Zahn mentioned above for an A type star; he too finds that the two convection zones are linked through penetrative motions.

\section{WORK IN PROGRESS}

Most of the recent results come from calculations that are based on the modal expansion procedure. It should not be forgotten, however, that in all cases the modal expansion was limited to only two, if not one, modes. The consequences of such a drastic truncation are not fully understood yet, and therefore these results should be used with some caution. It is true that most of these calculations have dealt with rather mild convection, involving convective fluxes of only a few percent. The feedback on the mean stratification is therefore very slight, and it is reasonable to assume that a multi-mode solution would not differ much from the superposition of an ensemble of single-mode solutions.

To check this point, it would be very worthwhile to undertake calculations with many more modes. This is now under its way thanks to the efforts of Nordlung, who will present some recent results in this colloquium, and to Marcus (1979). Marcus applies the modal expansions to a spherical geometry, and he just completed a first investigation of Boussinesq convection with as many as 15 modes. This enables him to calculate explicitly the motions on scales that are in the so-called inertial subrange, and which are fed only through the nonlinear interactions discussed earlier. 
For this reason, his results should be much less sensitive to the approximation used to close the system.

A less ambitious calculation, involving only three modes in its final form, is undertaken by Latour, Toomre and Zahn to describe the solar convection zone. Inspired by the observed scales separation, the modes are intended to represent respectively the granulation, the supergranulation, and the large cells which seem necessary to render the whole zone nearly adiabatic, even though they are not observed yet with certitude.

The same are also exmaining the penetration of convective motions into an adjacent stable layer, in the Boussinesq limit. Preliminary results have been reported by Zahn (1977): modal calculations with one or two hexagonal planforms predict a penetration which is of the order of the thickness of the unstable layer. Laboratory experiments with water around $0^{\circ} \mathrm{C}$ lead to the same conclusion.

An important effort is also spent to refine the treatment of radiative transfer in the presence of motions, in the optically thin limit. Legait and Gough (1979) compare various approximations with the exact solutions of the transfer equation, in the case of an anisotropic radiation field; their main goal is to test the validity of the Eddington approximation which is commonly used.

This list is certainly incomplete, but it has the merit to demonstrate that the subject of stellar convection is alive. There is good hope, therefore, that new and perhaps decisive progress will be reported when we meet the next time to discuss turbulence in stellar atmospheres. 


\section{REFERENCES}

Biermann, L. 1933, Z. Astrophys., 5, 117

Depassier, M.C., Spiegel, E.A. 1979 (in preparation)

Durney, B., Spruit, H. 1979 (preprint)

Gough, D.0. 1969, J. Atmosph. Sci., 26, 448

Gough, D.O., Moore, D.R., Spiegel, E.A., Weiss, N.0., Astrophys. J., 206, 536

Gough, D.0. 1978, Proc. EPS Workshop on Solar Rotation,87 (ed. Belevedere \& Paterno, Catania Univ. Press)

Knobloch, E. 1978, Astrophys. J., 225, 1050

Legait, A., Gough, D.0. 1979 (in preparation)

Maeder, A. 1975, Astron. Astrophys., 40, 303

Marcus, Ph. 1979 (in preparation)

Massaguer, J., Zahn, J.-P. 1979, Astron. Astrophys., (in press)

Nelson, G.D., Musman, S. 1977, Astrophys. J., 214, 912

Nelson, G.D. 1978a, Solar Phys., 60, 5

Nelson, G.D. 1978b, Ph. D. Thesis, Univ. of Washington

Öpik, E.J. 1950, M. N. Roy. Astron. Soc., 110, 559

Shaviv, G., Salpeter, E.E. 1973, Astrophys. J., 184, 191

Spiege1, E.A. 1965, Astrophys. J., 141, 1068

Spiegel, E.A. 1971, Ann. Rev. Astron. Astrophys., 9, 323

Toomre, J., Zahn, J.-P., Latour, J., Spiege1, E.A. 1976, Astrophys. J., 207, 545

Toomre, J., Gough, D.o., Spiege1, E.A. 1977, J. Fluid Mech., 79, 1

Toomre, J., Latour, J., Zahn, J.-P. 1977, BulZ. Americ. Astron. Soc., 9, 337

Unno, W., Spiege1, E.A. 1966, Pub. Astron. Soc. Japan, 18, 85

Vitense, E. 1953, 2. Astrophys., 32, 135

Zahn, J.-P. 1977, Problems of Stezzar Convection, 225 (ed. Spiegel \& Zahn, Lecture Notes in Physics, Springer) 